\title{
Harness Design and Coupling Stiffness for Two-Axis Torso Haptics
}

\author{
David I. Grow* \\ University of Utah \\ Department of Mechanical Engineering
}

\author{
John M. Hollerbach ${ }^{\dagger}$ \\ University of Utah \\ School of Computing
}

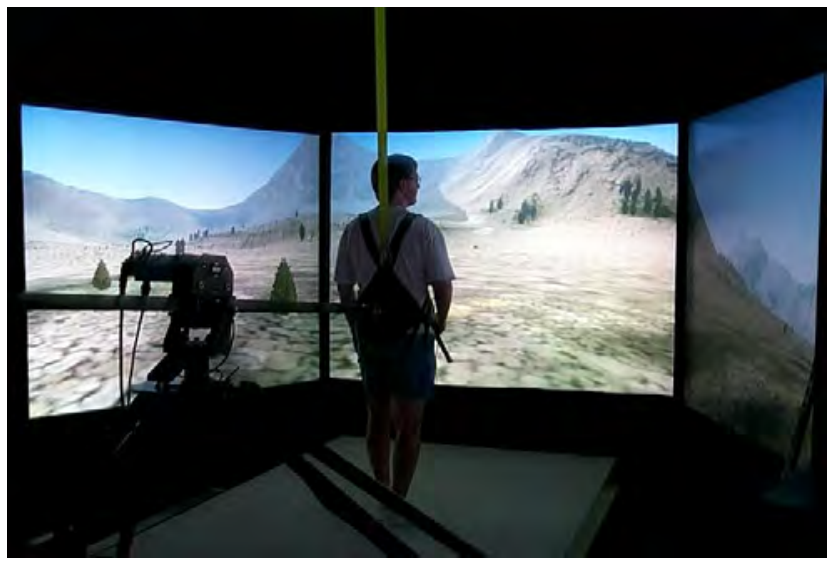

Figure 1: The mechanical tether of the Sarcos Treadport applies torso forces to a user via a harness worn by the user.

\section{Abstract}

This paper describes a harness design for transmitting both horizontal and vertical forces to the torso of a user on a locomotion interfaces. For horizontal forces, an exoskeleton-like mechanism distributes forces between the shoulders and hips and accommodates to the complicated motions of the back and shoulders relative to hips. For vertical forces, a body weight support harness is integrated into the exoskeleton-like mechanism. A passive elastic element has been devised that ensures consistent strap tightening. Measurements are presented that shows the stiffness of the mechanical coupling of harness to subject for the purposes of faithful force application to the torso.

CR Categories: H.5.2 [Information Interfaces and Presentation]: User Interfaces-Haptic I/O

Keywords: torso haptics, locomotion interfaces, harness design

\section{INTRODUCTION}

Previous research on the Sarcos Treadport locomotion interface has shown that forces applied to the torso of a user can be used for inertial force display [4] and for slope display [7, 14]. These studies have applied horizontal forces in the frontal direction with a 6-DOF mechanical tether that has an actuated prismatic joint (Figure 1). The tether also measures the user's position and orientation for belt speed control and directional heading [8].

In addition to frontal forces, there would be uses for torso forces in the side and vertical directions. In [6], a passive pulley-weight

\footnotetext{
*e-mail: david.grow@utah.edu

†e-mail:jhm@cs.utah.edu
}

Symposium on Haptic Interfaces for Virtual Environment and Teleoperator Systems 2006

March 25 - 26, Alexandria, Virginia, USA

1-4244-0226-3/06/\$20.00 C2006 IEEE system applying side forces was shown to simulate side slope walking reasonably well using a biomechanical analysis. Side forces would obviate the need to tilt the treadmill sideways [13], which few treadmills are designed to do and which complicates the mechanical design. Another reason for not tilting the treadmill, or at least adding torso forces in addition to tilt, is the higher bandwidth of torso force feedback which allows fast slope transients to be displayed. Vertical forces could be used to simulate walking in altered gravity environments [2], to provide partial weight support for rehabilitation purposes (e.g., the Lokomat System by Hocoma Inc.), and to simulate walking on steep frontal slopes.

With regard to simulating steep slopes, vertical support forces are required to balance the forces acting on a user. The forces on a person standing on an actual slope are (Figure 2):

$$
\begin{aligned}
F_{f} & =m g \sin \theta \\
F_{n} & =m g \cos \theta
\end{aligned}
$$

where $F_{f}$ is the force at the foot tangent to the slope, $F_{n}$ is the force at the foot normal to the slope, $m$ is the user's mass, $g$ is gravity, and $\theta$ is the slope angle. In the simulated slope case,

$$
\begin{aligned}
F_{f}^{\prime} & =F_{h} \\
F_{n}^{\prime} & =m g-F_{v}
\end{aligned}
$$

where $F_{f}^{\prime}$ is the foot force tangent to the level treadmill, $F_{n}^{\prime}$ is the foot force normal to the level treadmill, $F_{h}$ is the horizontal tether force, and $F_{v}$ is a possible vertical tether force. To make the experience feel the same to a user, the foot forces in the real versus simulated slopes should be the same:

$$
\begin{aligned}
& F_{f}^{\prime}=F_{f} \\
& F_{n}^{\prime}=F_{n}
\end{aligned}
$$

This means that

$$
\begin{aligned}
& F_{h}=m g \sin \theta \\
& F_{v}=m g(1-\cos \theta)
\end{aligned}
$$

In the diagram, the horizontal force $F_{h}$ makes the subject lean forward. The result should be that the ankle angle is the same as for real slope walking.

If there were no vertical support force $F_{v}$, the net force on the user in the simulated slope condition would be greater than the user's weight, because the user still feels his full weight on the horizontal belt and is in additional pulled by the tether. For small slopes, the difference is small; for example, at a 15 degree slope the net force is 1.03 times body weight. A 15 degree slope is about the steepest studied in [7, 14], and so not having a vertical tether force provides a negligible error. For steep slopes, the error is not negligible (e.g. roughly 1.13 times body weight at 30 degrees) and a vertical support force would have to be added. 


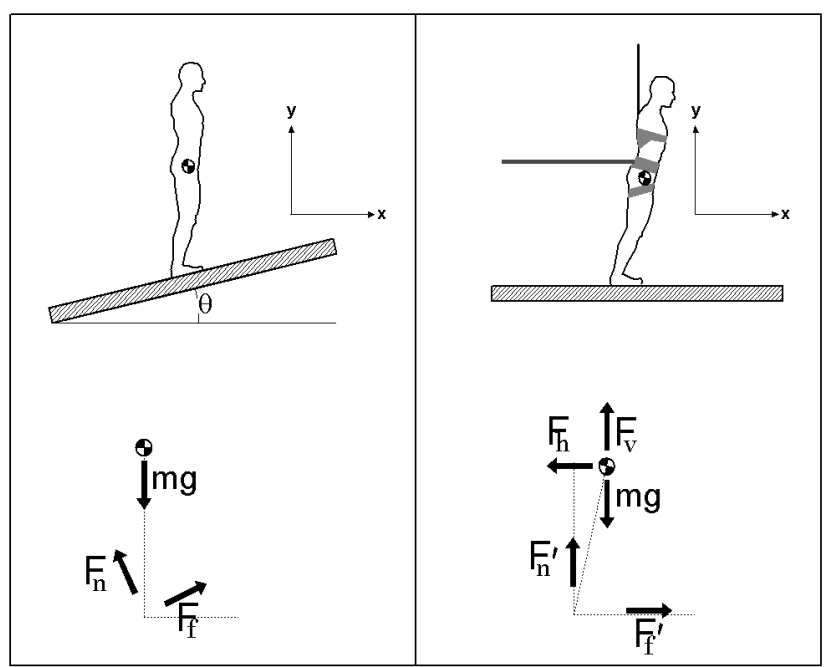

Figure 2: Slope Simulation Force

In this paper, we present a modified harness that both improves the display of frontal forces and supports vertical force display. The harness is required to display time varying forces in both axes comfortably for extended periods of time while not interfering with walking. This requires an orignal design as most harnesses support display of constant and/or single axis force [1, 5, 12]. These design constraints are satisfied through the combination of a prototype mechanism-based harness to support horizontal forces [3] with a vertical support harness inspired by harnesses developed for rehabilitation. Quantitative comparison is made on the mechanical coupling of the new harness to the body as compared to previous harness designs.

In all of these examples, there has to be some harness worn by the user to which the external force source is applied. When the forces are constant as in the passive application systems, the harness design can be simpler because the harness backlash need be taken up only at the beginning of force application. For time-varying force applications such as the simulation of varying slopes or changes in inertial force, any backlash or excess compliance will result in reduced force bandwidth, fidelity, and stability.

\section{Previous Harness Designs}

Harness designs for the Treadport have to date been devised only to transmit frontal forces. Initially harnesses were devised that used soft straps and that borrowed from backpack harness designs (Figure 3(A)). Fabric panels cover most of the chest and back with straps between. In the front, the top panel routes the shoulder straps to a waist panel and is needed to hold the shoulder straps away from the neck. The waist panel contains a plastic plate, and was suggested by a backpack maker to relieve weight from the shoulder straps by pushing on the plastic plate with the stomach muscles. There is also a hip strap which goes around the bony part of the hips and gives about the best mechanical coupling of any part of the harness. There are leg loops to prevent the harness from riding up and to support a user who falls by connection of the harness to a safety strap in the ceiling.

In the rear panel a curved metal plate is embedded for fit to users' backs and for connection to the tether. There is a convergence of straps from the front to the fabric cover for the metal plate: the shoulder straps, straps from the top and waist panels, the hip strap, and the leg loops. The backpack-style harness allows adaptability to a variety of users and is comfortable during prolonged use.

There are several drawbacks to this design. The first is that asymmetry exists in axial force display. When the tether presses against the user's back, the compliance is small. However, when the tether is pulled, the back of the harness deflects until the user's torso is compressed enough to provide the target reaction force. There is substantial backlash and low stiffness which makes closed-loop force control with the tether extremely difficult. The backlash and stiffness are affected by how tightly the straps are adjusted, but there is no method to regulate strap tightness to guarantee consistency. The force distribution between the straps and different parts of the torso is fixed but unknown; it is suspected that the force distribution between shoulders and hips is a key determiner of torso lean [3]. The strap system does not adjust to the complex degrees of freedom of the back, such as elongation when bending or twisting. As a subject bends or twists, the strap system must adjust by moving on the body in some uncontrolled fashion and tightening at some straps while loosening at others.

To overcome some of the limitations of the backpack-style harness, a mechanism-based prototype harness was created [3]. The back side of the harness is composed of aluminum tubing and metal plate systems at the hips and at the shoulders (Figure 3(B)). The shoulder plate system contains various adjustments for differentsized users. A spherical joint at the back of the hip and two revolute pairs allow torso flexion in the sagittal plane. Several straps fix the mechanism to the user's body. This harness greatly improved the coupling of the tether to the subject torso. Moreover, the tether could attach to the rigid spine at several different heights, which was shown to affect the force distribution between shoulders and hips and the amount of torso lean [3]. Regulating torso lean may be important in the simulation of frontal slopes, since generally speaking subjects lean less than on real slopes [14]. Limitations to the prototype design included a fitting time of up to five minutes, a lack of flexibility to fit small or large users, and a lack of the degree of freedom (DOF) required for the user to bend in the coronal plane.

Neither this mechanism-based prototype nor the backpack-style harness was designed to support vertical forces comfortably over an extended period of time. There are a number of commercial harness types to support vertical forces. One type of harness is designed especially for dynamic events, such as rock climbing and industrial harnesses, by lifting largely at the hip. Another type, Bodyweight Support (BWS) harnesses, is used for physical rehabilitation or over-speed training. They are designed to apply a static upward force by lifting both with leg loops and with a torso vest. The vest lifts the chest both by virtue of grabbing under the rib cage and friction; to increase friction, the vest covers most of the chest surface.

\section{New HaRness Design}

A new harness has been designed, that combines an improved mechanism-based harness with a BWS harness. Starting first with just the mechanism portion (Figures $3(\mathrm{C})$ and 4), an upper-back plate and a lower-back plate are connected by a telescoping spine. The lower-back plate is fixed to the spine, while the upper-back plate is connected to the spine via a spherical joint. The result is that the upper and lower plates can move independently and allow all necessary DOFs of the back including coronal flexion. The lower plate is curved to match the contour of the subject's lower back and to increase the moment of inertia. The upper portion of the spine couples to the fall arrestor. The rear of the spine has an integrated rail so that the tether mount position can be varied.

The spine's prismatic joint is nominally perpendicular to the tether. This ensures that tether force does not apply compression or tension to the user's torso. The spherical joint mounted on the upper plate is as close as possible to the plate without hitting a user's back 

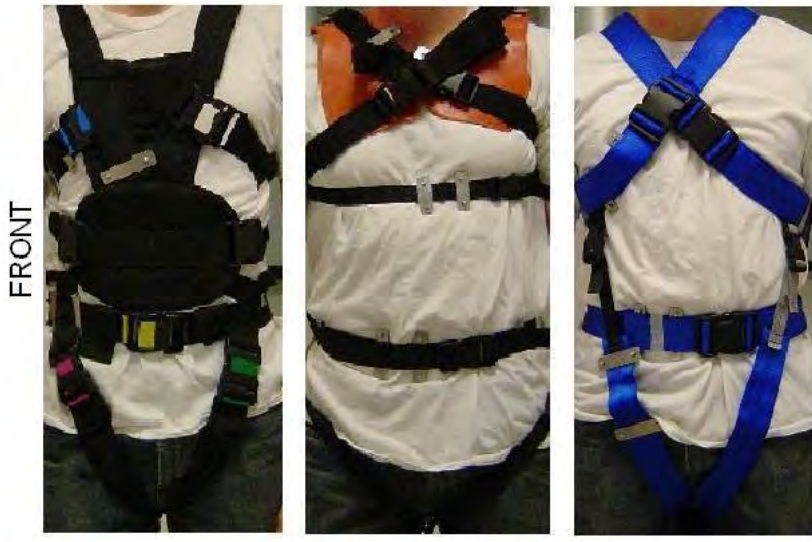

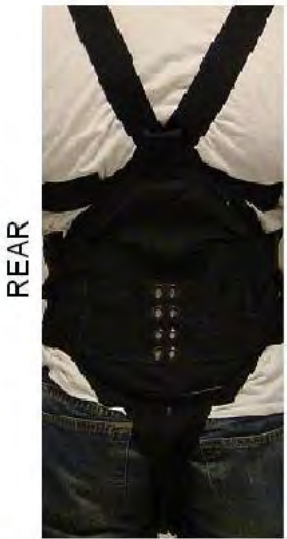

(A)

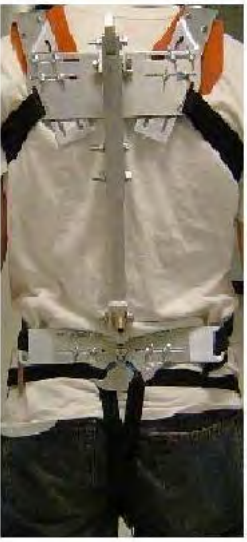

(B)

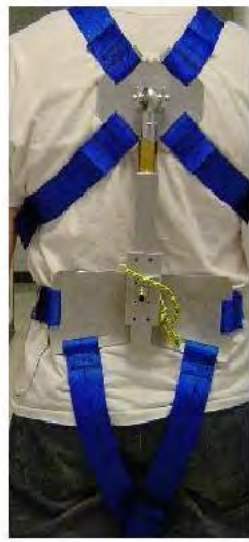

(C)
Figure 3: (A) Backpack style harness. (B) Mechanism-based harness version 1 and $(C)$ version 2 .
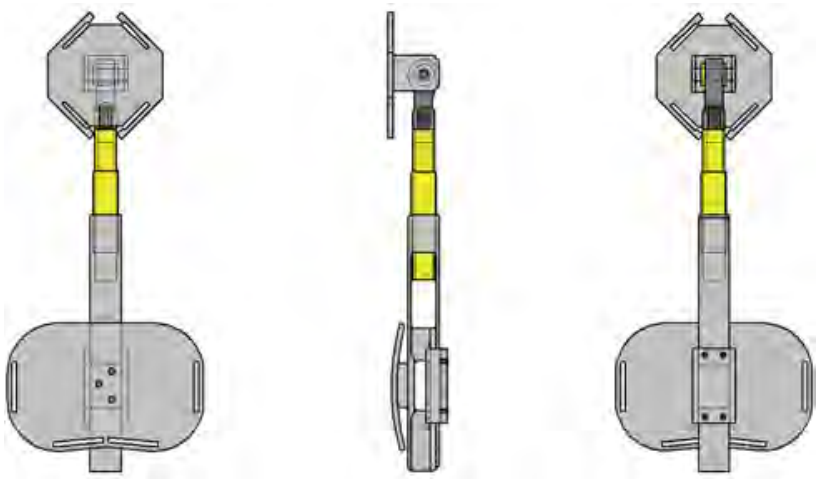

Figure 4: Schematic of the mechanism-based harness version 2. when curved, in order to minimize a twisting torque by the tether on a user turned somewhat to the side. The prismatic joint has $10 \mathrm{~cm}$ of passive travel to allow the user to bend naturally in the sagittal plane and to adjust automatically to users of different height; users from 5'2" to 6'2" can be accomodated. As with the backpack style harness, fitting times for the new harness were under a minute.

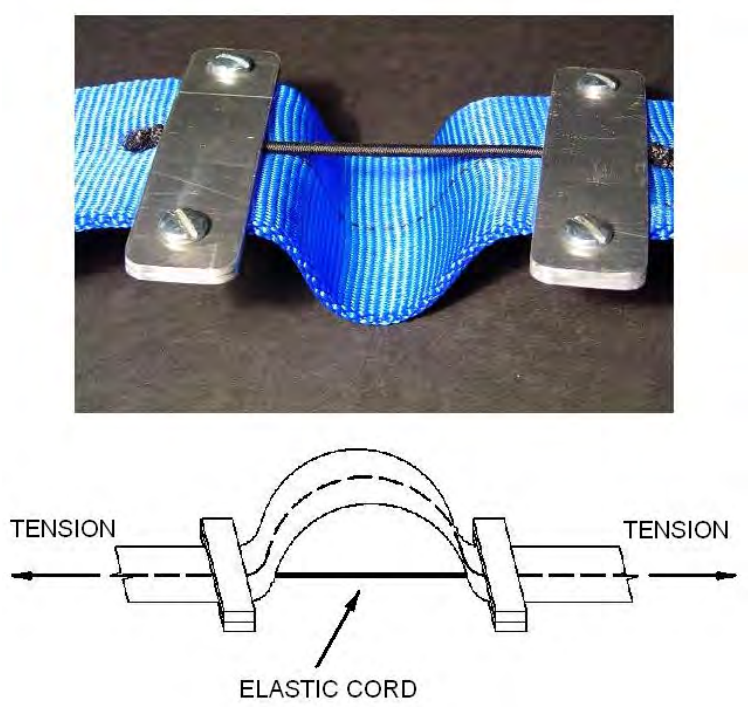

Figure 5: Tension gauges.

Strap tension has a large effect on the effective stiffness of the harness-user system. To ensure that the strap tension is consistent for various harnesses and subjects, simple tension gauges were constructed and installed in parallel with each of the harness straps. An elastic element was set such that the strap had the appropriate tension when the slack in the strap was taken up. Tensions of five pounds for the belt strap of each harness and two pounds for all other straps were used (see Figure 5).

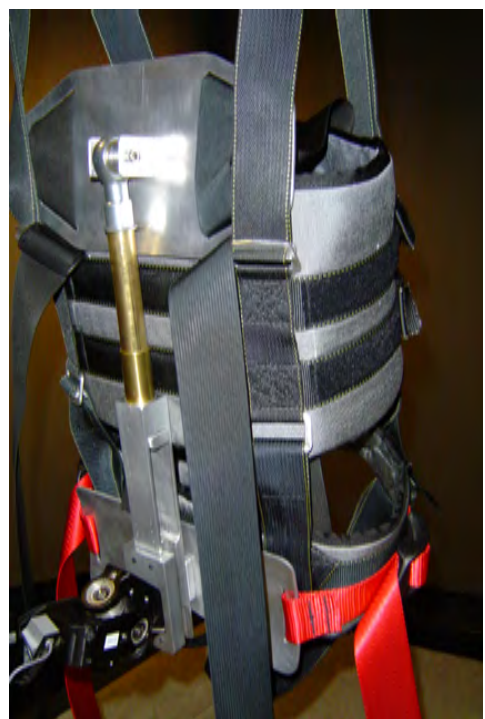

Figure 6: BWS harness integrated with the mechanism-based harness.

Extended vertical force display during locomotion requires that the harness distribute force over the upper and lower torso. 
This necessitates a departure from the strap routing used in the mechanism-based harnesses shown in Figure 3. Rolling straps directly around the shoulder applies pressure to the subclavian artery and vein reducing circulation to the arms [3]. Instead crossing the straps over the chest fails for extended vertical support because for some subjects, the criss-cross straps creep upward and put pressure on the neck.

A variety of commercial BWS harnesses were tested and demonstrated merit for both applying the required lift at the upper torso and for supplying the necessary rigidity in the horizontal direction. The key difference is that these harnesses incorporate straps that go circumferentially around the torso. These straps are integrated into a vest to increase the contact area. The vest remains below the nipple line to avoid the aforementioned issues. A local manufacturer produced a modified BWS harness that allowed the integration of the new mechanism (Figure 6). The resulting harness successfully permits comfortable force application in the target axis while satisfying the other design requirements.

\section{Stiffness Evaluation}

For the BWS harness, force application is meant to be unilateral: body weight is supported by pulling up. Downward forces to simulate increased loads are not planned at the present time. For the mechanism portion of the new harness, forces are designed to be bilateral: the harness may push as well as pull. Because of the afore-mentioned backlash problems with the backpack-style harness, a key design goal of the mechanism-based harness is to reduce backlash and increase the stiffness of the harness/user system. To evaluate the mechanical coupling of the harnesses depicted in Figure 3, stiffness measurements were made both on a rigid mannikin CementMan (Figure 7) and on users wearing the harnesses.

CementMan was created to investigate the stiffness of the harness strap systems alone. A torso form was filled with cement and the harnesses were strapped tightly around it. Compliance was then measured by recording the deflection under the application of a known force. The mode of flexure was also observed. Push and pull forces of $90 \mathrm{~N}$ were applied using a load cell and the deflection was measured at the top, bottom, and center of each harness. The data for each harness was averaged. The effective spring constants for the backpack, prototype, and new harnesses were 10, 60, and $370 \mathrm{~N} / \mathrm{mm}$, respectively (Figure 8). The rear of the backpack style harness experienced global deformation, while the flexure observed in the prototype occurred primarily in the lower-back plate. The deflection that occured with the new harness was so small that it was difficult to determine the source. Both mechanism based harnesses showed substantial improvement over the backpack style harness. The improved stiffness of the new harness was due to the use of a curved lower back plate.

Next, the effective spring constants of the user/harness systems were measured. Six subjects, four men and two women, aged 24-48 wore each of the three harnesses while force was applied. An openloop system drove the tether force sinusoidally with a frequency of $0.2 \mathrm{~Hz}$ and amplitudes of 30,60, 90, and $120 \mathrm{~N}$. The subjects stood on the stationary belt and braced themselves by holding a rail. An Optotrak system was employed to record the position of markers attached near the subjects COM and on the tether. The projection of distance between the user COM and an arbitrary location on the tether was projected onto the tether axis. The change in this distance as a function of time was used to determine the effective compliance of the harness/user system.

The harness/user stiffnesses with the backpack, prototype, and new harnesses were 8.6, 12.9, and $13.9 \mathrm{~N} / \mathrm{mm}$, respectively (Figure 9). Clearly the compliance of the user begins to dominate in the case of the mechanism based harnesses. Nonetheless, the mechanism based harnesses led to at least a 50\% improvement in system

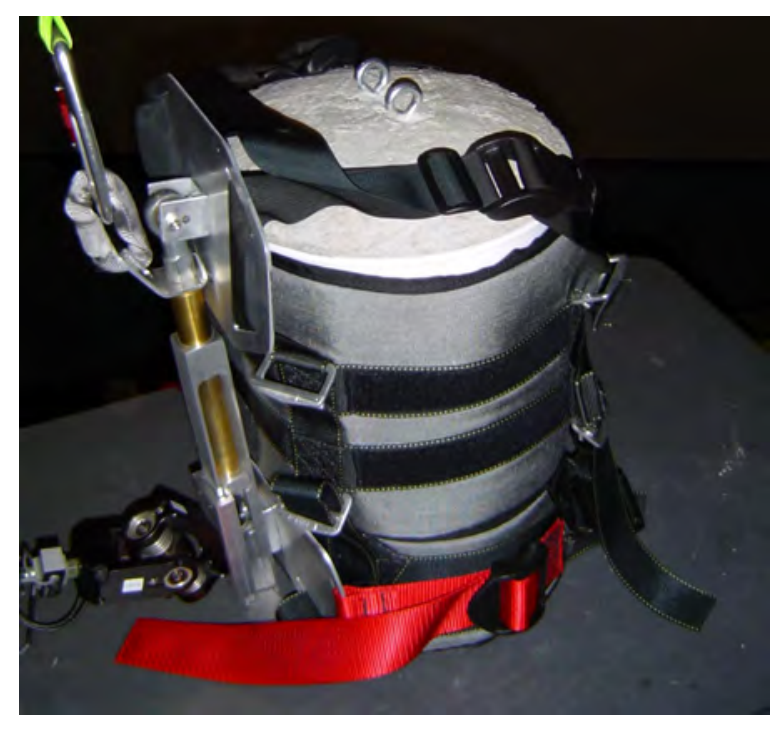

Figure 7: CementMan wearing the mechanism-based harness version

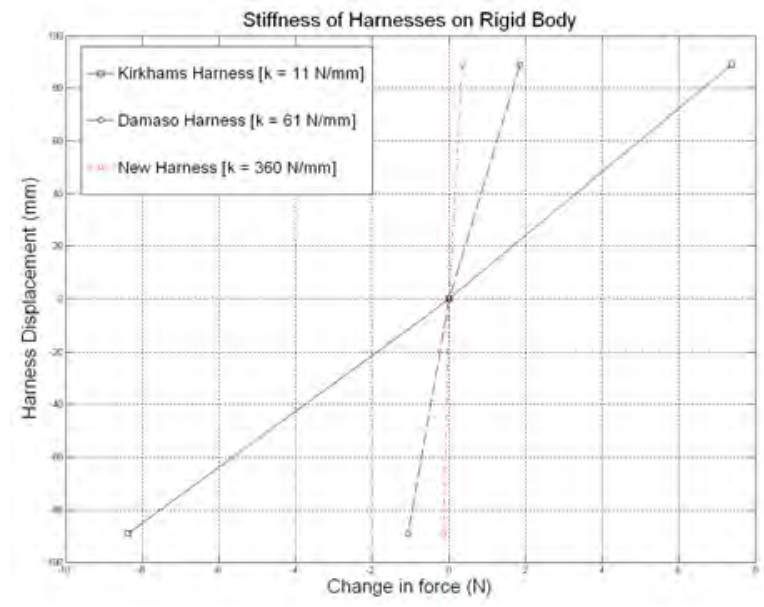

Figure 8: Harness/CementMan stiffness results. 
stiffness. It is also significant to note the variance in system stiffness by subject. For each harness, the values found differ by a factor of two, roughly. For the subjects tested, both gender and body mass index correlate to system stiffness.

\section{Stiffness for Backpack-Style Harness}

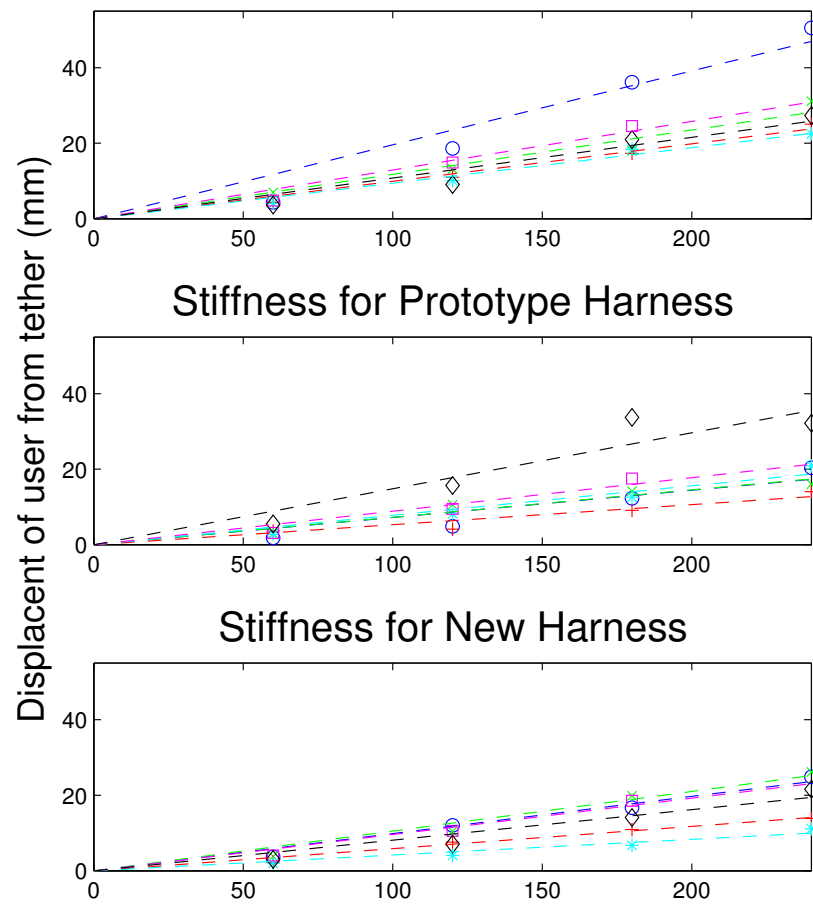

Average Stiffness for Harnesses

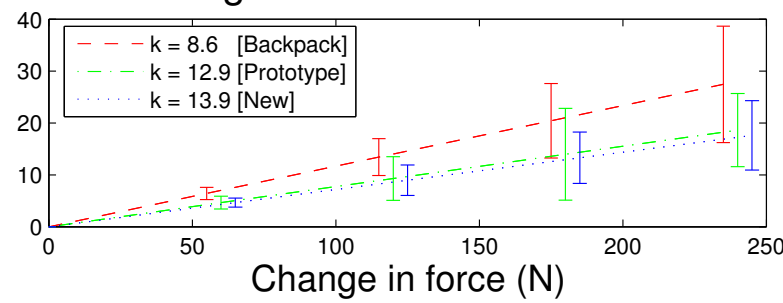

Figure 9: User/harness stiffness results (six subjects in each of three harnesses).

\section{Acknowledgements}

This research was supported by NSF grants IIS-0113996 and IIS0428856

\section{REFERENCES}

[1] Y. Chang, C. Hmerski, and R. Kram. Applied horizontal force increases impact loading in reduced gravity running. In J. Biomechanics, pages 675-685, 2001.

[2] Y.H. Chang, H.W.C. Huang, C.M. Hamerski, and R. Kram. The independent effects of gravity and inertia on running mechanics. J. Experimental Biology, 203:229-238, 2000.

[3] D. Checcacci, J.M. Hollerbach, Ryan Hayward, and M. Bergamasco. Design and analysis of a harness for torso force application in locomotion interfaces. In Eurohaptics, pages 53-67, July 6-9, 2003.

[4] R.R. Christensen, J.M. Hollerbach, Y. Xu, and S.G. Meek. Inertialforce feedback for the treadport locomotion interface. Presence: Teleoperators and Virtual Environments, 9:1-14, 2000.

[5] T. Griffin, N. Tolani, and R. Kram. Walking in simulated reduced gravity: mechanical energy fluctuations and exchange. In J. Applied Physiology, pages 383-390, 1999.

[6] J.M. Hollerbach, D. Checcacci, H. Noma, Y. Yanagida, and N. Tetsutani. Simulating side slopes on locomotion interfaces using torso forces. In Proc. 11th Symposium on Haptic Interfaces for Virtual Environments and Teleoperation, pages 91-98, March 22-23, 2003.

[7] J.M. Hollerbach, R. Mills, D. Tristano, R.R. Christensen, W.B. Thompson, and Y. Xu. Torso force feedback realistically simulates slope on treadmill-style locomotion interfaces. Intl. J. Robotics Research, 12:939-952, 2001

[8] J.M. Hollerbach, Y. Xu, R. Christensen, and S.C. Jacobsen. Design specifications for the second generation sarcos treadport locomotion interface. In Proc. ASME Dynamic Systems and Control Division, volume DSC-Vol. 69-2, pages 1293-1298, March 22-23, 2000.

[9] John M. Hollerbach. Locomotion interfaces. In K.M. Stanney, editor, Handbook of Virtual Environments: Design, Implementation, and Applications, pages 239-254. Lawrence Erlbaum Associates, Inc., 2002.

[10] L.A. Jones, M. Nakamura, and B. Lockyer. Development of a tactile vest. In Proc. 12th Symposium on Haptic Interfaces for Virtual Environments and Teleoperation, pages 82-89, 2004.

[11] R. Kirkman and M. Metzger. Conceptual design of an adaptive wind tunnel for the generation of unsteady complex flow patterns. In ASME 2005 Fluids Engineering Division Summer Meeting and Exhibition, June 19-23, 2005.

[12] J. McCrory and et al. Evaluation of a treadmill with vibration and stabilization (tvis) for use on the international space station. In $J$ Applied Biomechanics, volume 15, pages 292-302, 1999.

[13] H. Noma and T. Miyasato. Design for locomotion interface in a large scale virtual environment. atlas: Atr locomotion interface for active self motion. In Proc. ASME Dynamic Systems and Control Division, volume DSC-Vol. 64, pages 111-118, 1998.

[14] C. Parker, D. Carrier, and J.M. Hollerbach. Validation of torso force feeback slope simulation through an energy cost comparison. In World Haptics Conference, pages 446-451, March 18-20, 2005.

\section{Discussion}

The new harness better couples the user to the force-feedback device to allow higher force bandwidth and support of multi-axial force display. Other features include adaptability, quick fitting time, and comfort. Currently, work is underway to actuate the harness in the vertical direction. The ability to regulate torso lean and apply vertical force may improve slope display. The effect of these tactics will be measured empirically. 